\title{
Type 1 Diabetes Mellitus reversal via implantation of magnetically purified microencapsulated pseudoislets
}

A. Espona-Noguera ${ }^{1,2 \dagger}$, J. Etxebarria-Elezgarai ${ }^{4 \dagger}$, L. Saenz del Burgo ${ }^{1,2}$, A. CañibanoHernández $^{1,2}$, H. Gurruchaga ${ }^{1,2}$, F.J. Blanco ${ }^{7,8}$, G. Orive ${ }^{1,2,3}$, Rosa M. Hernández ${ }^{1,2}$, F. Benito-Lopez ${ }^{5}$, J. Ciriza ${ }^{1,2}$, L. Basabe-Desmonts $^{4,6} *$ and J.L. Pedraz ${ }^{1,2 *}$

${ }^{1}$ NanoBioCel Group, Laboratory of Pharmaceutics, School of Pharmacy, University of the Basque Country (UPV/EHU), Paseo de la Universidad 7, 01006, Vitoria-Gasteiz, Spain.

${ }^{2}$ Biomedical Research Networking Center in Bioengineering, Biomaterials, and Nanomedicine (CIBER-BBN), Vitoria-Gasteiz, Spain.

${ }^{3}$ University Institute for Regenerative Medicine and Oral Implantology - UIRMI (UPV/EHUFundación Eduardo Anitua); BTI Biotechnology Institute, Vitoria-Gasteiz, Spain.

${ }^{4}$ BIOMICs-microfluidics Research Group, Microfluidics Cluster UPV/EHU, University of the Basque Country, Spain.

${ }^{5}$ AMMa LOAC Research Group, Microfluidics Cluster UPV/EHU, University of the Basque Country, Spain

${ }^{6}$ Basque Foundation of Science, IKERBASQUE, Spain

${ }^{7}$ INIBIC-Hospital Universitario La Coruña, La Coruña, Spain

${ }^{8}$ Biomedical Research Networking Center in Bioengineering, Biomaterials and Nanomedicine (CIBER-BBN), La Coruña, Spain

†These authors contributed equally to this work

* Corresponding authors

E-mail address: joseluis.pedraz@ehu.eus lourdes.basabe@ehu.eus 


\section{ABSTRACT}

Microencapsulation of pancreatic islets for the treatment of Type I Diabetes Mellitus (T1DM) generates a high quantity of empty microcapsules, resulting in high therapeutic graft volumes that can enhance the host's immune response. We report a 3D printed microfluidic magnetic sorting device for microcapsules purification with the objective to reduce the number of empty microcapsules prior transplantation. In this study, INS1E pseudoislets were microencapsulated within alginate (A) and alginate-poly-L-lysine-alginate (APA) microcapsules and purified through the microfluidic device. APA microcapsules demonstrated higher mechanical integrity and stability than A microcapsules, showing better pseudoislets viability and biological function. Importantly, we obtained a reduction of the graft volume of $77.5 \%$ for A microcapsules and $78.6 \%$ for APA microcapsules. After subcutaneous implantation of induced diabetic Wistar rats with magnetically purified APA microencapsulated pseudoislets, blood glucose levels were restored into normoglycemia $(<200 \mathrm{mg} / \mathrm{dL})$ for almost 17 weeks. In conclusion, our described microfluidic magnetic sorting device represents a great alternative approach for the graft volume reduction of microencapsulated pseudoislets and its application in T1DM disease.

KEYWORDS: microcapsule sorting, alginate, superparamagnetic iron oxide nanoparticles, microfluidics, Type I Diabetes Mellitus 


\section{Introduction}

Type 1 Diabetes Mellitus (T1DM) is a metabolic disorder characterized by the autoimmune destruction of the pancreatic $\beta$-cells and, subsequently, an absolute deficiency of insulin to maintain blood-glucose homeostasis (de Groot, Schuurs et al., 2004; Yun Lee, Hee Nam et al., 2007). Currently, exogenous insulin injection is widely implemented being the most effective therapy. However, administration of insulin is onerous for the patients, since it is difficult for these formulations to avoid both hyperglycemia and hypoglycemia episodes, which can lead to diabetic complications (Skrzypek, Groot Nibbelink et al., 2017; Wenjuan Li, Ruxing Zhao et al., 2014). Alternatively, the Edmonton protocol emerged as a promising method to restore the endogenous $\beta$-cell function, thus, normalizing the glucose metabolic control in patients with T1DM (Street, Lakey et al., 2004). This procedure is based on the transplantation of isolated cadaveric pancreatic islets, thus providing a new $\beta$-cell source capable of assessing blood-glucose levels and secrete insulin in a glucose-dependent manner in T1DM patients. Although great successes have been achieved in the glucose homeostasis restoration, there are still several issues to overcome before the widespread clinical application. One of the main obstacle of islet transplantation is the long-term use of immunosuppressants to avoid the immune rejection of transplanted islets (Edmond A. Ryan, Jonathan R.T. Lakey et al., 2001; Ryan, Lakey et al., 2002; Wenjuan Li et al., 2014). In order to circumvent this problem, pancreatic islets can be immunoisolated by microencapsulation techniques within a biocompatible matrix (Brian Buder, Michael Alexander et al., 2013; Paul de Vos, Marchetti, 2002).

The microencapsulation technology provides a physical barrier between the therapeutic cells and the host immune system, thus avoiding the entrance of high molecular weight immune components such as immunoglobulins and immune cells (El-Sherbiny, Yacoub, 2013). Moreover, the structure of the microcapsule permits the diffusion of nutrients and 
oxygen between the environment and the core of the microcapsule, while allowing the release of the therapeutic molecules produced by the embedded cells, as for example, insulin (Yang, Yoon, 2015). Among different biomaterials, such as poly(hydroxyethyl methacrylate-methyl methacrylate), agarose, chitosan, and polyethylene glycol (PEG); alginate is the most commonly used biomaterial in pancreatic islet microencapsulation (Borg, Bonifacio, 2011). This natural polymer has excellent properties for biomedical applications as it demonstrates high biocompatibility and low toxicity (Lee, Mooney, 2011; Sakata, Sumi et al., 2012). Moreover, alginate microcapsules can be modified to tune their physical properties, like their mechanical and diffusion properties, which are critical to ensure their integrity and to allow the release of therapeutic molecules produced by the encapsulated cells, respectively (Smidsrod, Skjak-Braek, 1990; Strand, Ryan et al., 2001). However, this technology has several technical obstacles that difficult its clinical application. One crucial problem is the high number of empty microcapsules generated during the islet microencapsulation process, leading to a high graft volume, which can enhance the host immune reaction after implantation (Kobayashi, Aomatsu et al., 2006). Although the reduction of the graft volume is nowadays still being accomplished by separating the microencapsulated islets from the empty microcapsules by hand selection, the manual procedure is tedious, slow, and complicates its reproducibility (Brian Buder et al., 2013; King, 2001; Park, Lee et al., 2017; Wanyu Chen, Mark Lisowski et al., 2012). On this regard, many microfluidic techniques for cell sorting have been proposed over the last decade, including active and passive sorting; the former being mostly employed (Wyatt Shields IV, Reyes et al., 2015; Xi, Zheng et al., 2017). Active sorting can be categorized, according to the actuation mechanism, as electric, acoustic, magnetic, pneumatic and thermal sorting (Adeyemi, 2017; Ahne Myklatun, Michele Cappetta et al., 2017; Girault, Kim et al., 2017; Wyatt Shields IV et al., 2015). Among all, magnetic actuation is the most commonly used method in many applications (Wyatt Shields IV et al., 
2015; Xi et al., 2017). Different applications in which magnetic separation techniques and microfluidic devices have been implemented, in macro or mesoscale systems, including the extraction and concentration of magnetized porcine pancreatic islets from the digested pancreas that are previously magnetized in vivo (Kennedy, Todd et al., 2007), or the microencapsulation of pancreatic islets or other cells within microfluidic devices (Tendulkar, Mirmalek-Sani et al., 2012). However, this application has not been used yet for the magnetic purification of magnetized microencapsulated islets from empty microcapsules. Overall, microfluidics systems constitute microscale platforms that enable the automatization and monitorization of the purification process. Importantly, these systems also minimize technical errors improving the reproducibility of the purification process. Moreover, as described by Temiz et al., the 3D printing technology enables the fabrication of complex microfluidic devices in a single-step, and allows the prototyping and low volume production of monolithic LOC devices for microfluidic applications, that do not require an additional sealing or microfluidic port integration step (Temiz, Lovchik et al., 2015). This facilitates the design conformation for the inlet/outlet connectors, as well as the integration of other external components (e.g., magnets) with no need of any external packaging. Furthermore, 3D printing enables easy modifications of design features, accelerating the optimization stage of the microfluidic performance.

In this manuscript, with the aim of reducing the therapeutic graft volume in T1DM, we report a 3D printed magnetic sorting microfluidic device for the purification of microencapsulated pseudoislets. To this end, we combined the superparamagnetic iron oxide nanoparticles (SPIONs) and the microfluidic technologies. On the one hand, SPIONs provide magnetic properties to the pseudoislet-containing microcapsules that allow their separation, and, on the other hand, microfluidics offers the creation of a platform at microscale level that enables the purification process, its automatization and monitorization. For the validation of 
the device, we generated a pancreatic islet-like cell aggregates from the INS1E rat insulinoma cell line. After purifying the microencapsulated pseudoislets through the microfluidic device, different parameters were evaluated in vitro such as the viability, metabolic activity, insulin production and mechanical integrity of the purified and non-purified microcapsules. Then, the therapeutic potential of purified microencapsulated rat pancreatic pseudoislets was investigated in Wistar rats with induced T1DM.

\section{Materials and Methods}

\subsection{Materials and reagents}

Ultrapure low-viscosity high guluronic acid sodium alginate $(\mathrm{G} / \mathrm{M}$ ratio $\geq 1.5)$ with a molecular weight of 75-200kDa was purchased from FMC Biopolymer (Sandvika, Norway), penicillin/streptomycin/glutamine (P/S/G) from Invitrogen (Carlsbad, United States), HEPES buffer from Lonza (Basilea, Switzerland), trisodium citrate dihydrate and sodium chloride $(\mathrm{NaCl})$ from Panreac (Castellar del Vallès, Spain), and Rat Insulin ELISA kit from Mercodia (Uppsala, Sweden). Poly-L-lysine hydrobromide (PLL, 15-30kDa), poly(ethyleneimine) solution (PEI), sodium pyruvate, $\beta$-mercaptoethanol, citric acid solution, the Cell Counting Kit 8 (CCK-8), potassium chloride $(\mathrm{KCl})$, magnesium chloride $\left(\mathrm{MgCl}_{2}\right)$, calcium chloride $\left(\mathrm{CaCl}_{2}\right)$, bovine serum albumin (BSA), streptozotocin (STZ) and D-glucose were purchased from Sigma-Aldrich (San Luis, United States). Dulbecco's Modified Eagle's Medium (DMEM), Roswell Park Memorial Institute (RPMI) medium 1640, fetal bovine serum (FBS), penicillin-streptomycin (P/S) and LIVE/DEAD ${ }^{\circledR}$ Viability/Cytotoxicity Kit were purchased from Life Technologies (Carlsbad, United States).

\subsection{Cell culture and INS1E pseudoislets formation}

D1 mouse mesenchymal stem cells (D1-MSCs) (ATCC, USA) and engineered D1MSCs to express the green fluorescence protein (GFP) (D1-MSCs-GFP) were grown in 
DMEM high glucose medium supplemented with $10 \%$ FBS and $1 \%$ of P/S as previously described (Megías, Arco et al., 2017). Rat insulinoma INS1E cells provided by the University of Geneva Medical Center (Merglen, Theander et al., 2004), were cultured in complete medium consisting of RPMI 1640 supplemented with $10 \%$ FBS, 1\% P/S/G, $1 \%$ sodium pyruvate $100 \mathrm{mM}, 1 \mathrm{M}$ HEPES buffer and $0.1 \%$ mercaptoethanol. The INS1E cell line was used for pseudoislets formation by the hanging-drop method. Briefly, cells were trypsinized to obtain a cell suspension of $2.5 \times 10^{4}$ cells $/ \mathrm{mL}$, and $20 \mu \mathrm{L}$ droplets, containing 500 cells/droplet, were applied onto the lid of a $245 \times 245 \mathrm{~mm}$ cell culture dish (Corning Incorporated, New York, United States). The lid was carefully flipped and placed onto the dish, which had been previously filled with distilled water to maintain humidity. Cells were cultured for five days to allow pseudoislet formation. Next, pseudoislets were harvested and transferred into a non-adherent $60 \mathrm{~mm}$ culture dish (Corning Incorporated, New York, United States). All cells and pseudoislets were cultured in a humidified atmosphere containing 5\% $\mathrm{CO}_{2}$ at $37^{\circ} \mathrm{C}$.

\subsection{Synthesis of SPIONs and cell magnetization}

The SPIONs used in this study were nanoparticles (NPs) of $\mathrm{Fe}_{3} \mathrm{O}_{4} / \mathrm{PEI}$ that were prepared by chemical co-precipitation as described by Munoz de Escalona, et al. (Muñoz de Escalona, Sáez-Fernández et al., 2016). Briefly, $\mathrm{Fe}_{3} \mathrm{O}_{4} \mathrm{NPs}$ were re-dispersed in a $0.1 \mathrm{~N}$ citric acid solution, sonicated for 40 minutes and, finally, the dispersion was adjusted to $\mathrm{pH} 7$ with 0.5M NaOH. After that, SPIONs were coated by adding a PEI aqueous solution drop-wise to the iron oxide aqueous dispersion $\left(3: 4\right.$ ratio of $\left.\mathrm{PEI}: \mathrm{Fe}_{3} \mathrm{O}_{4}\right)$ under mechanical stirring (2000 rpm). Then, the dispersion was neutralized again to $\mathrm{pH} 7$ with $0.5 \mathrm{M} \mathrm{HCl}$, and the NPs were magnetically washed by repeated separation from the liquid medium by a permanent $0.4 \mathrm{~T}$ magnet. Finally, NPs were resuspended in distilled water. 
D1-MSCs-GFP were magnetized as described by Megías, et al. (Megías et al., 2017). Briefly, NPs were diluted in complete culture medium and $10 \mathrm{~mL}$ added to a confluent T75 flask at a $11 \mu \mathrm{g}$ of NPs $/ 10^{5}$ cells ratio. Then, the flask was placed onto a $0.4 \mathrm{~T}$ magnet for 15 minutes. Next, cells were detached and microencapsulated. On the other hand, INS1E pseudoislets in suspension were placed in a $60 \mathrm{~mm}$ culture dish with complete medium containing optimal NPs concentration and incubated for 24 hours to magnetize the pseudoislets.

\subsection{Microencapsulation}

For microencapsulation, sterile $1.5 \%(\mathrm{w} / \mathrm{v})$ sodium alginate solution was prepared in a $1 \%(\mathrm{w} / \mathrm{v})$ mannitol solution. Then, it was filtered through a $0.22 \mu \mathrm{m}$ pore Minisart Syringe Filter (Sartorius, Gotinga, Germany). Afterward, cells were suspended in the alginate solution at a cell density of $5 \times 10^{6}$ cells / $\mathrm{mL}$ or 2000 pseudoislets $/ \mathrm{mL}$. These suspensions were extruded in an electrostatic droplet generator (Nisco Engineering, Duluth, United States) through a $0.17 \mathrm{~mm}$ inner diameter needle using a $10 \mathrm{~mL}$ sterile syringe with a peristaltic pump at $5.9 \mathrm{~mL} / \mathrm{h}$ flow rate. Microcapsules were collected in a $55 \mathrm{mM} \mathrm{CaCl}_{2}$ bath and maintained in agitation for 10 minutes to obtain the alginate (A) microcapsules. Next, microcapsules were chemically crosslinked with $0.05 \%$ (w/v) PLL for 5 minutes, and then, they were coated with $0.1 \%(\mathrm{w} / \mathrm{v}$ ) alginate for 5 minutes, giving rise to alginate-poly-L-lysine-alginate (APA) microcapsules. All procedures were performed at room temperature, under aseptic conditions. Microcapsules were cultured in the correspondent complete medium at $37{ }^{\circ} \mathrm{C}$ in a $5 \% \mathrm{CO}_{2}$ atmosphere.

\subsection{Fabrication of the cell sorting microfluidic device}

The magnetic purification device was designed using PTC Creo Parametric 3D modeling Software and manufactured in a Formlab stereolithography 3D printer (Formlabs, 
Somerville, United States) using an optically clear acrylic material, Clear resin FLGP CL02

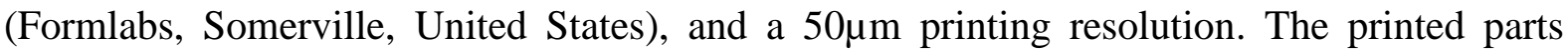
were rinsed in an isopropanol bath for $4 \mathrm{~min}$ to eliminate the excess of uncured resin, and subsequently, post-cured under $365 \mathrm{~nm}$ UV light for 15 minutes to ensure complete polymerization and reach the highest strength and stability for the parts. Supports were removed using a snip, and the parts were gently sand polished to assure transparent and highquality polymeric parts. Finally, the chip was finalized by covering the top channel with a pressure sensitive PSA AR-MH-92712 adhesive (Adhesive Research, Limerick, Ireland). Figure 2a exhibits the finalized microfluidic magnetic cell sorting device for purification of the magnetized-microcapsules. The device integrates commercial neodymium magnets of 1.3T (Supermagnete, Gottmadingen, Germany) with a magnetic clamping force of $10.8 \mathrm{~N}$ for the $5 \mathrm{~mm} \times 5 \mathrm{~mm}$ magnet, and $6.86 \mathrm{~N}$ for the $5 \mathrm{~mm}$ diameter $\mathrm{x} 3 \mathrm{~mm}$ height magnet. Thereby, while the magnetized capsules are envisioned to move to the upper channel due to the magnetic field, the empty capsules will be divided equally between both channels, leading to a separation of non-magnetized capsules that allows recovery of highly concentrated magnetized samples in the upper outlet.

\subsection{Setup for microfluidic microcapsules sorting}

The characterization of microcapsules purification was carried out with A microencapsulated non-magnetized D1-MSCs (non-mag-D1-MSCs) and magnetized D1MSCs-GFP (mag-D1-MSCs-GFP). Both types of microcapsules were mixed at different mag-D1-MSCs-GFP/non-mag-D1-MSCs ratios $(5 / 95,10 / 90,25 / 75,50 / 50,75 / 25)$ to evaluate the purification performance at each condition. The setup for the characterization of the purification involved: 1) a positive pressure flow controller (Fluigent MFCS ${ }^{\mathrm{TM}}$ FLEX) to drive the liquid through the microfluidic device; 2) a microscope and 3) a fluorescent reader (FluoroReader®, Elveflow) to analyze the distribution of the mag-D1-MSCs-GFP 
microcapsules in situ (Figure 1a). Fluorescence at the outlet of the channels was displayed in real time in order to monitor the deviation of mag-D1-MSCs-GFP from the purification channel. Additionally, the purification efficiency for different conditions was quantified by flow cytometry. Briefly, non-purified and purified microcapsules from each condition were dissolved in $1 \%$ trisodium citrate dihydrate. Then, cells were collected by centrifugation, rinsed with DPBS, and transferred to a FACS tube. The proportion of mag-D1-MSCs-GFP and non-mag-D1-MSCs after purification was analyzed for all samples using the BD FACS Calibur flow cytometer (BD Bioscience, Franklin Lakes, United States). Three independent experiments, with three replicates each one, were conducted.

(a)

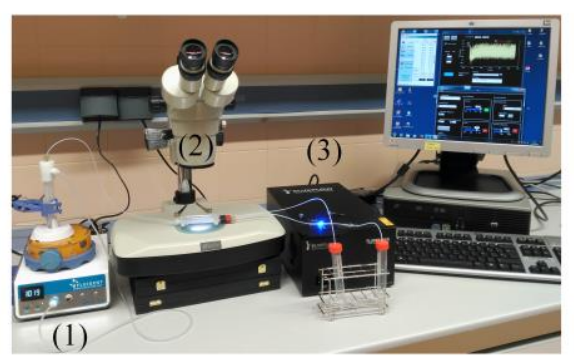

(b)

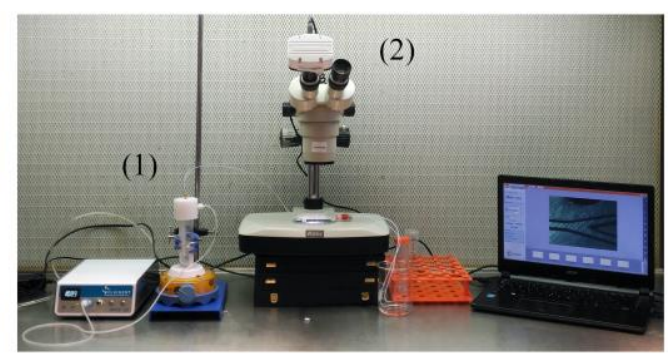

Figure 1. (a) Experimental setup for the optimization of microfluidic magnetic cell sorting flow conditions comprised of a positive pressure flow controller (1), a microscope with an integrated camera (2) and a fluorescent reader (3). (b) Experimental setup for microfluidic magnetic purification of INS1E, in sterile conditions, comprised of a positive pressure flow controller (1) and a microscope with an integrated camera (2).

The purification of A and APA microencapsulated INS1E pseudoislets was carried out under sterile conditions by placing the setup within a laminar flow cabinet after ethanol and UV light sterilization. The setup was similar than the one used in the separation of non-magD1-MSCs and mag-D1-MSCs-GFP; but instead of a fluorescent reader, a microscope with an integrated camera (ISH500 Tucsen Photonics) and a TCapture software application (ISCapture, Tucsen Photonics) were used for real-time monitorization of the purification process (Figure 1b). 
Also, the purification efficiency after separation was determined by measuring the 24 hours secreted insulin from the non-purified and purified samples. Briefly, $50 \mu \mathrm{L}$ of microcapsules from each sample were rinsed twice with medium, resuspended in $0.5 \mathrm{~mL}$ of medium, and incubated for $24 \mathrm{~h}$ at $37^{\circ} \mathrm{C}$ and $5 \% \mathrm{CO} 2$. The insulin content of collected supernatants was quantified with the Rat Insulin ELISA kit following the manufacturer's recommendations. Three independent experiments, with three replicates each one, were conducted.

\subsection{Cell metabolic activity and viability determination}

Metabolic activity was determined using the Cell Counting Kit 8 (CCK-8). During the optimization of the pseudoislets magnetization process, 50 magnetized pseudoislets were harvest after 24 hours of incubation with different NPs concentrations (5, 10, 20, 40, 80, 160, 320 and $640 \mu \mathrm{g}$ of $\mathrm{NPs} / \mathrm{mL}$ ), and resuspended in $500 \mu \mathrm{L}$ of complete medium containing $50 \mu \mathrm{L}$ of CCK-8 reagent, placed in a $500 \mu \mathrm{L}$ conical tube and incubated in a shaker for 4 hours at $37^{\circ} \mathrm{C}$ into the incubator. The supernatants were collected, transferred into a 96 well-plate, and the absorbance was read out on an Infinite M200 TECAN plate reader (TECAN Trading AG, Männedorf, Switzerland) at 450nm with a reference wavelength set at $650 \mathrm{~nm}$. Control tests were carried out similarly incubating the pseudoislets in complete medium without NPs. Three independent tests were conducted for each condition.

To study the metabolic activity of the purified microencapsulated pseudoislets, $50 \mu \mathrm{L}$ of microcapsules were resuspended in $150 \mu \mathrm{L}$ of complete medium with $15 \mu \mathrm{L}$ of $\mathrm{CCK}-8$ reagent, plated in a 96-well plate, incubated, and the absorbance was read following the same

procedure previously described. Non-purified encapsulated pseudoislets were used as controls. Three independent tests were conducted for each condition. 
On the other hand, cell viability was determined by fluorescence microscopy, and structural integrity of the microcapsules was determined by bright field microscopy. With this aim, $25 \mu \mathrm{L}$ of purified encapsulated pseudoislets were stained with the LIVE/DEAD ${ }^{\circledR}$ Viability/Cytotoxicity Kit. First, the microcapsules were washed twice with DPBS. Then, they were resuspended in $200 \mu \mathrm{L}$ of $0.5 \mu \mathrm{M}$ calcein $\mathrm{AM}$, and $0.5 \mu \mathrm{M}$ ethidium homodimer- 1 in DPBS and, finally, microcapsules were transferred into 96-well plates and incubated at room temperature for 40 minutes in the dark. Next, samples were observed under a Nikon TMS microscope with the excitation/emission settings for calcein AM staining (495/515nm) and ethidium homodimer staining $(495 / 635 \mathrm{~nm})$. The images of fluorescence and brightfield microscopy were acquired with a Nikon Eclipse TE2000-Scamera (Nikon, Amsterdam, Netherlands), which was controlled by the EclipseNet software version 1.20.0, and at least three independent experiments were analyzed.

\subsection{Glucose-Stimulated Insulin Secretion}

In order to assess the microencapsulated pseudoislets insulin secretory capacity, the Glucose-Stimulated Insulin Secretion assay (GSIS) was performed 24 hours after magnetic purification. $50 \mu \mathrm{L}$ of purified and non-purified microcapsules were washed four times with Krebs-Ringer bicarbonate (KRB) buffer composed of $125 \mathrm{mM} \mathrm{NaCl}, 3 \mathrm{mM} \mathrm{KCl}, 0.85 \mathrm{mM}$ $\mathrm{CaCl}_{2}, 1.3 \mathrm{mM} \mathrm{MgCl}_{2}, 0.1 \%$ BSA and 25mM HEPES buffer. After washing, microcapsules were incubated at $37^{\circ} \mathrm{C}$ in the incubator in $\mathrm{KRB}$ buffer for 1 hour with shaking. Next, KRB buffer was replaced with $\mathrm{KRB}$ containing $3.3 \mathrm{mM}$ glucose and incubated for 2 hours (low glucose condition). Supernatants were collected, and the samples were washed with KRB four times again. Next, they were incubated for 2 hours in KRB containing $16.6 \mathrm{mM}$ glucose (high glucose condition), and supernatants were collected.

The insulin secretion for 24 hours from culture supernatants was determined at days 1 , 7, 21 and 28 after magnetic separation. At each timepoint, 50 $\mu \mathrm{L}$ of microcapsules were rinsed 
twice with medium, resuspended in $0.5 \mathrm{~mL}$ of medium, and incubated for $24 \mathrm{~h}$ at $37^{\circ} \mathrm{C}$ and $5 \%$ $\mathrm{CO}_{2}$. Then, supernatants were collected. The insulin content of collected supernatants was quantified with the Rat Insulin ELISA kit following the manufacturer's recommendations. Three independent experiments, with three replicates each one, were conducted.

\subsection{Induction of Diabetes in vivo and implantation of pseudoislets-containing microcapsules}

Male Wistar rats of 150-200g from ENVIGO (Sant Feliu de Codines, Spain) were housed with sterile food and autoclaved water. Six days before implantation of microencapsulated pseudoislets, diabetes was induced by a single intravenous injection of $80 \mathrm{mg} / \mathrm{kg}$ body weight of STZ diluted in $50 \mathrm{mM}$ sodium citrate buffer. Animals were considered diabetic if blood glucose exceeded $20 \mathrm{mmol} / \mathrm{L}(>360 \mathrm{mg} / \mathrm{dL})$ for at least two consecutive measurements. Then, diabetic rats were divided into 4 groups. The first group was implanted subcutaneously with $0.4 \mathrm{~mL}$ from the purified microcapsules pool, containing 3000 microencapsulated equivalent pseudoislets/rat, suspended in $1 \mathrm{~mL}$ PBS using an 18gauge catheter; the second group was implanted with the same volume of microcapsules from the non-purified pool; the third group received the same volume of empty microcapsules (without cells), and in the fourth group (negative control) diabetic animals were not implanted. Non-diabetic rats were monitored in parallel as controls of glycemia too. During implantation, animals were maintained under anesthesia by isoflurane inhalation. Blood samples were collected from the tail vein to measure blood glucose levels with a glucometer (Abbott Laboratories, Chicago, United States), during the first week every 24 hours and afterward, weekly. Animals were also weighted daily during the first week after implantation, then twice per week and, at the end of the study, weekly. All the experimental procedures were performed in compliance with protocols approved by the institutional animal care and 
use committee of the University of Basque Country UPV/EHU (Permit Number: M20_2016_082_CIRIZA ASTRAIN).

\subsection{Glucose tolerance test}

A glucose tolerance test (GTT) was performed two months after microcapsules implantation. A dose of $2 \mathrm{~g}$ glucose $/ \mathrm{kg}$ bodyweight was administered intraperitoneally to rats after 12 hours fasting, and blood glucose levels were measured at 0, 0.5, 1, 1.5, 2, 2.5, 3, 4, 5 6 and 7 hours after glucose challenge using a glucometer.

\subsection{Immunohistochemistry}

Animals from each group were sacrificed by $\mathrm{CO}_{2}$ inhalation and, the implants were retrieved and fixed with $4 \%$ formaldehyde (Panreac, Castellar del Vallès, Spain) for histological analyses. Serial horizontal cryostat sections $(14 \mu \mathrm{m})$ were processed for hematoxylin and eosin or Masson's trichrome (H\&E) staining. Photographic images were taken using a Nikon D-60. Microscopy sections were examined by an expert pathologist blinded to the treatments. The presence and distribution of infiltrating cells, and preservation of the tissue along with the extension of fibrosis were evaluated.

\subsection{Statistical analysis}

Statistical analysis was performed with SPSS software, version 21.00.1. Data were expressed as means \pm standard deviation, and differences were considered significant for comparison of groups using ANOVA, Tukey's Post Hoc Test when $p<0.05$ after assessing their normal distribution. Kaplan-Meier cumulative survival analysis was used to determine the animal survival of each group of study after transplantation. 


\section{Results and discussion}

\subsection{Optimization of microfluidic device design and flow conditions for microcapsules purification}

The microfluidic pathway within the device consisted in a "Y" shape configuration, a main channel $36 \mathrm{~mm}$ long which split in two channels $37 \mathrm{~mm}$ long. The design integrated commercial neodymium magnets located in parallel to the main channel close to the bifurcation in order to trigger the movement of the magnetized capsules to the upper channel due to the magnetic field. The empty capsules were expected to be divided equally between both channels, leading to a separation of non-magnetized capsules that allows recovery of highly concentrated magnetized samples in the upper outlet (Figure 2a-b).

(a)

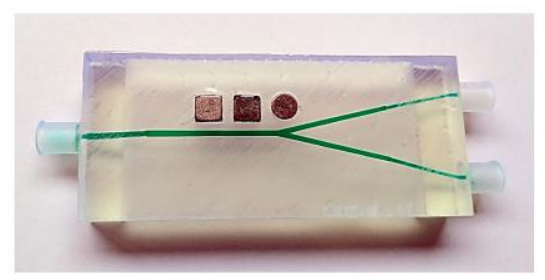

(b)

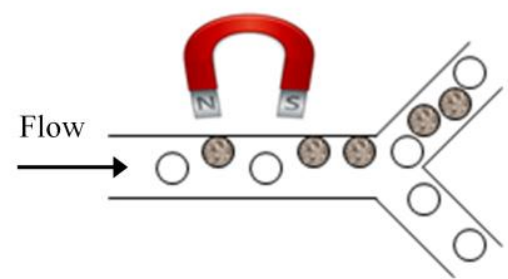

Figure 2. (a) 3D printed microfluidic chip sealed with a pressure sensitive adhesive, integrating commercial magnets. The chip has been filled with green-colored solution to highlight the internal channels. (b) Working principle of the magnetic separation of the microcapsules: while the magnetized-microcapsules move to the upper channel due to the force generated by the magnet, the empty capsules divide equally between both channels.

For setting up the magnetic sorting device and the purification performance, D1-MSCsGFP cells were magnetized (mag-D1-MSCs-GFP), while D1-MSCs cells were not magnetized (non-mag-D1-MSCs), next generating microcapsules from both populations, representing microcapsules containing islets and empty microcapsules.

Different parameters were studied, such as distinct device architectures and magnet configurations. Variations on the microfluidic channel dimensions and the position of the connectors for the inlet and outlet tubings were analyzed, as well as the magnets 
configuration, the use of different fluid pressure conditions and distinct microcapsules concentrations. Initially, the connectors and tubings were arranged perpendicularly to the main microfluidic channel (Figure 3a), which resulted in changes in the direction of the flow due to elbows inside the microchannels, that influenced on the local resistance and generated sudden local velocity decrease, decelerating and accumulating the microcapsules at a certain point, and causing clogging issues. This problem was resolved when the connectors were arranged in parallel to the main fluidic channel (Figure 3b). Similar effects were observed by Li et al. (Li, Tian et al., 2011), who investigated the flow performance of a cell suspension near the chip inlet area and compared the different performances when using vertical and parallel inlet connectors. Vertical inlet connectors comprised larger dead volumes and initial impact driven by the vertical direction of hydrodynamic force, resulting in flow irregularities near the inlet area and formation of cell blockages, which is especially detrimental to experiments that require recovery of purified cells. In contrast, parallel inlets alleviated channel blockage caused by large dead volume and irregular flow directions (Li et al., 2011). The parallel configuration of the connectors was possible due to the fabrication flexibility provided by the $3 \mathrm{D}$ printing technique in comparison with more traditional fabrication techniques to generate microfluidic devices such as photolithography or PDMS casting (Ho, $\mathrm{Ng}$ et al., 2015). 
(a)

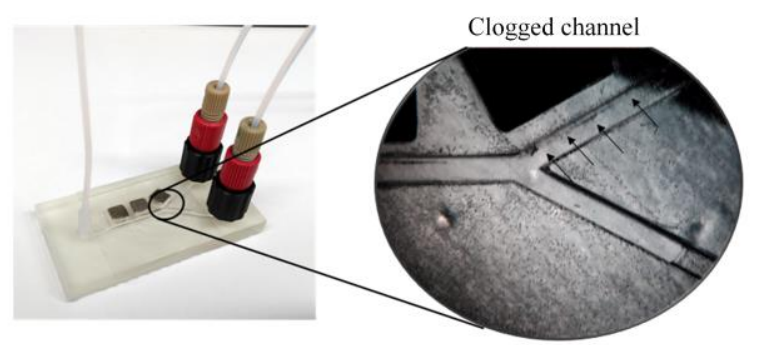

(b)

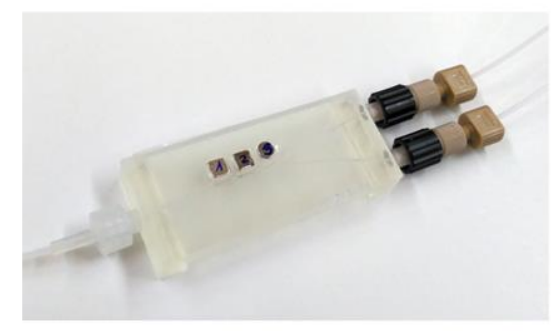

Figure 3. Optimization of the design and architecture of the magnetic separation device. (a) Image of a microfluidic sorting device prototype with perpendicular inlets and outlets connectors clogged by microcapsules. (b) Final 3D printed microfluidic sorting device with the optimal inlets and outlets connectors design, magnets positioning and configuration avoiding microcapsules clogging. Black arrows: microcapsules clogging the microchannel.

Regarding the dimensions of the microchannels, the best flow containing microcapsules through the microfluidic device, with no clogging issues, was obtained when using the main channel of $1 \mathrm{~mm} \times 1 \mathrm{~mm}$ cross-section, which splits in two channels of $750 \mu \mathrm{m} \times 750 \mu \mathrm{m}$ cross section (Table 1). In this way, alginate microcapsules with an average diameter of $450 \mu \mathrm{m}$ were satisfactorily driven through the microchannels. In addition, a balance between the magnetic field and the flow velocity inside the microchannels was essential to provide a highthroughput purification system and increase the purification efficiency and yield. Different shapes and number of magnets were analyzed for various fluidic pressures (Table 1). On the one hand, employing inappropriate balances of too high magnetic forces and too low fluid velocities, in general, led to the retention of the magnetized microcapsules near the bifurcation of the split channels, clogging the outlet channel and the whole purification. On the other hand, low magnetic forces required low flow rates to ensure successful separation of magnetized microcapsules, but this led to a reduced movement of the microcapsules after the 
bifurcation, clogging the microchannels. For this reason, the optimal configuration consisted of three magnets; two with rectangular shape $(5 \mathrm{~mm} \times 5 \mathrm{~mm} \times 5 \mathrm{~mm})$ and a smaller circular magnet $(5 \mathrm{~mm}$ diameter and $3 \mathrm{~mm}$ height), generating a total magnetic clamping force of around $28.5 \mathrm{~N}$. For this magnetic force, fluid velocities at 50, 60, and 75mbar fluidic pressures, led to the retention of the magnetized microcapsules near bifurcation of the split channels, while higher fluidic pressures (200mbar) resulted in accumulation of the capsules in the constriction of the outlet channels, again clogging the whole microfluidic system, resulting 100mbar the optimal fluidic pressure.

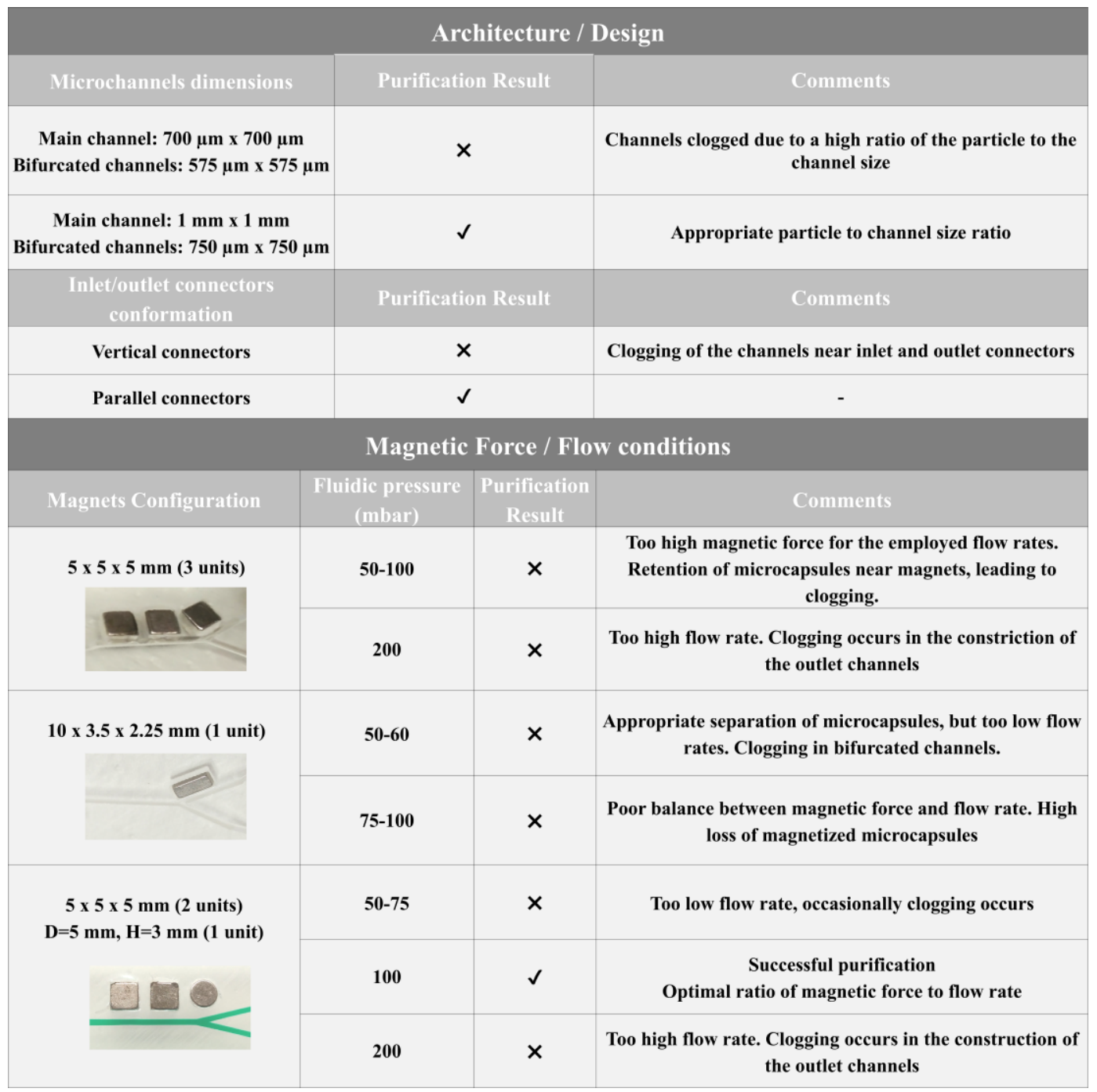

Table 1. Experimental results obtained during the optimization of the purification system design and operation conditions. 
When the design of the microfluidic device was optimized, we proceeded to optimize the particle concentration for its purification through the device. According to Dresaire et al., the clogging dynamic is controlled by the concentration of large particles and the flow rate in the channel. In case of high flow rates, for example, clogging of a channel can be caused by the simultaneous arrival of particles that plug the cross-section of the channel, typically at the inlet/outlet or at a constriction. The clogging probability increases with the particle concentration, with the flow rate and with the ratio of the particle to the channel size (Dressaire, Sauret, 2016).

Different microcapsules suspensions were prepared diluting different amounts of microcapsules suspensions in $30 \mathrm{~mL}$ of cell culture media. Dilutions of $5,2,1,0.5 \mathrm{~mL}$ of microcapsules $/ 30 \mathrm{~mL}$ of media were prepared, resulting in final capsules concentrations of $465 \cdot 10^{3}, 186 \cdot 10^{3}, 93 \cdot 10^{3}$ and $46 \cdot 10^{3}$ microcapsules $/ \mathrm{mL}$ respectively. Those samples were processed through the microfluidic device and then analyzed. Microcapsules concentrations higher than $93 \cdot 10^{3}$ microcapsules $/ \mathrm{mL}$ resulted in the accumulation of microcapsules in the inlets of the microfluidic device, thus blocking the sample flow. However, at 1/30 and 0.5/30 $\mathrm{mL}$ of microcapsules/mL of media, microcapsules were able to flow through the microfluidic channels while the generated magnetic field promoted the separation of the mag-D1-MSCsGFP from non-mag-D1-MSCs microcapsules, attracting the mag-D1-MSCs-GFP microcapsules towards the top outlet channel, while splitting equally towards both outlet channels the non-mag-D1-MSCs or empty microcapsules (supplementary material, Video 1 and 2).

Hence, the final microfluidic device design consisted in a main channel of $1 \mathrm{~mm} \mathrm{x}$ $1 \mathrm{~mm}$ cross-section, which split in two channels of $750 \mu \mathrm{m} \times 750 \mu \mathrm{m}$ cross section where the inlet and outlet connectors were arranged in parallel to main fluidic channel; a magnet configuration of three magnets strategically placed alongside the main microfluidic channel, 
two with rectangular shape $(5 \mathrm{~mm} \times 5 \mathrm{~mm} \times 5 \mathrm{~mm})$ and an smaller circular magnet $(5 \mathrm{~mm}$ diameter and $3 \mathrm{~mm}$ height) placed near the bifurcation; and using a pressure of 100mbar imposed across the whole system, which generated a flow rate of $1.3 \mathrm{~mL} / \mathrm{min}$ with a microcapsules dilution of $1 \mathrm{~mL}$ of microcapsules $/ 30 \mathrm{~mL}$ of media.

\subsection{Characterization of the magnetic purification performance}

Once the optimal device design and working conditions were determined, the performance of the purification system depending on the ratio of the magnetized microcapsules respect to the non-magnetized was studied. Different mag-D1-MSCsGFP/non-mag-D1-MSCs ratios were evaluated (5/95, 10/90, 25/75, 50/50, 75/25) with special attention to the lower ratios 5/95 and 10/90, which are similar to the pancreatic isletscontaining microcapsules/empty microcapsules proportion after microencapsulation of real islets in preclinical studies.

First, the green fluorescence from mag-D1-MSCs-GFP microcapsules at the outlet of the channels was displayed, in situ and in real-time, monitoring the loss of magnetizedmicrocapsules that diverted from the purification channel. The fluorescent readouts from both outlet channels showed that most of the mag-D1-MSCs-GFP microcapsules were attracted towards the magnets and driven through the top channel (Figure 4a), and a few of them were diverted towards the bottom channel (Figure 4b). 
(a)

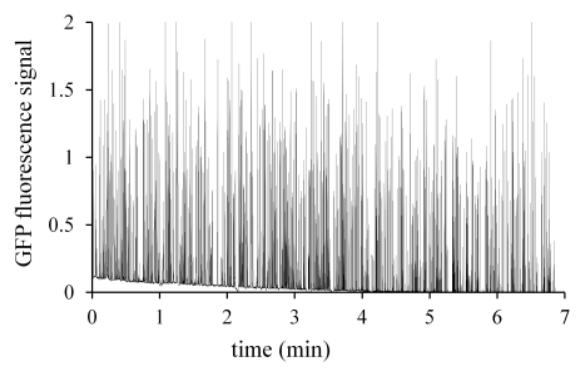

(c)

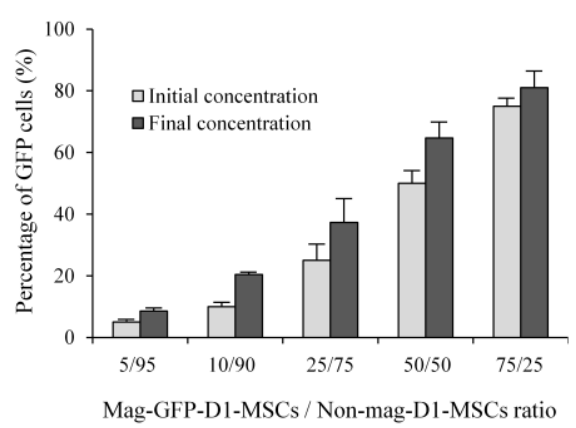

(b)

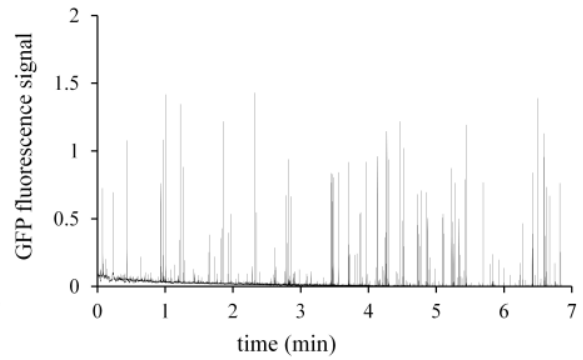

(d)

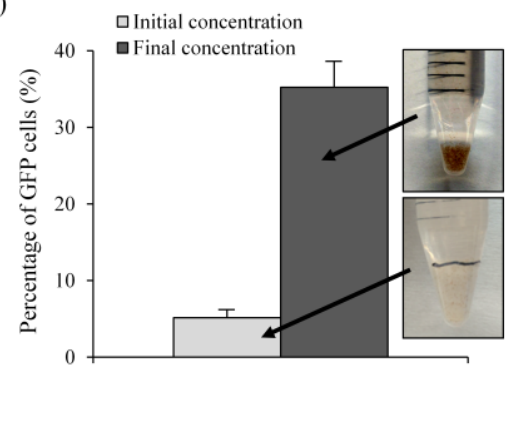

Figure 4. Optimization of the purification flow conditions for the microfluidic magnetic cell sorting, using magD1-MSCs-GFP and non-Mag-D1-MSCs microcapsules. (a) Fluorescent readouts obtained for the top and, (b) bottom outlet tubings. (c) Flow cytometry analysis of non-purified and purified microcapsules from different samples mixtures at different mag-D1-MSCs-GFP/non-mag-D1-MSCs ratios. (d) Flow cytometry analysis of the non-purified and purified microcapsules after 3cycles through the sorting device, for a sample with a 5/95 mag-D1-MSCs-GFP/non-mag-D1-MSCs ratio.

Second, the non-purified samples and the purified microcapsules collected from the magnet channel were quantified by flow cytometry, for each concentration ratio, in order to determine the efficiency of the purification. Flow cytometry results showed that, after the purification, the concentration of the mag-D1-MSCs-GFP microcapsules increased for all the suspensions isolated from the top channel in comparison with the non-purified samples (Figure 4c). The increase in the mag-D1-MSCs-GFP concentration in the different mag-D1MSCs-GFP/non-mag-D1-MSCs ratios relies on the elimination of the non-mag-D1-MSCs microcapsules during the purification step. Thereby, the concentration of the mag-D1-MSCsGFP microcapsules was highly increased for the lowest ratios, due to a larger amount of empty microcapsules, achieving an increase of mag-D1-MSCs-GFP percentage from $5.1 \pm 0.36 \%$ to $8.6 \pm 1.02 \%$, which supposed a purification yield of $80 \pm 7.1 \%$ for the $5 / 95$ ratio 
sample. In contrast, the concentration increase was not so prominent for high initial ratios, obtaining a purification yield of $33.3 \pm 3.95 \%$ for the $75 / 25$ ratio sample.

Finally, a sample with an initial ratio of 5/95 mag-D1-MSCs-GFP / non-mag-D1-MSCs microcapsules, envisioned to mimic a real scenario of pancreatic islets-containing microcapsules together with a huge number of empty microcapsules, was successively circularized three times through the magnetic sorting device. The mag-D1-MSCs-GFP concentration was considerably increased, from $5.14 \pm 1.01 \%$ to $35.23 \pm 3.4 \%$, thus obtaining a highly concentrated mag-D1-MSCs-GFP microcapsules pool with $6.91 \pm 0.55$ times more presence of mag-GFP-D1-MSCs in the purified sample compared to the non-purified sample (Figure 4d). Therefore, in this case, a theoretical implantation volume of $10 \mathrm{~mL}$ on microcapsules could be reduced 6.91 times to a final implantation volume of $1.48 \mathrm{~mL}$, which would mean an implantation volume reduction of $85.2 \%$.

\subsection{Determination of the optimal conditions for pseudoislets magnetization}

The preservation of pseudoislet viability and the conferring of magnetic motion after magnetization are crucial factors for future in vivo studies and clinical applications. For that reason, we evaluated the toxic and motion effect of $\mathrm{Fe}_{3} \mathrm{O}_{4} / \mathrm{PEI}$ NPs concentration on INS1E pseudoislets after magnetization. To that end, pseudoislets were exposed to different $\mathrm{Fe}_{3} \mathrm{O}_{4} / \mathrm{PEI}$ NPs concentrations $(0,5,10,20,40,80,160,320$ and $640 \mu \mathrm{g} / \mathrm{mL})$ for 24 hours. No significant influence on pseudoislet metabolic activity was detected after exposing to 5, 10, 20, 40 and $80 \mu \mathrm{g} \quad \mathrm{Fe}_{3} \mathrm{O}_{4} / \mathrm{PEI} \mathrm{NPs} / \mathrm{mL}$ conditions, compared to non-magnetized pseudoislets used as controls (Figure 5). However, at higher concentrations, a dose-dependent cytotoxicity was observed with a significant reduction in the metabolic activity of pseudoislets of $30.9 \%$ for $160 \mu \mathrm{g} \mathrm{Fe}_{3} \mathrm{O}_{4} / \mathrm{PEI} \mathrm{NPs} / \mathrm{mL}$, $80.6 \%$ for $320 \mu \mathrm{g} \mathrm{Fe}_{3} \mathrm{O}_{4} / \mathrm{PEI} \mathrm{NPs} / \mathrm{mL}$ and, $87.5 \%$ for $640 \mu \mathrm{g} \mathrm{Fe}_{3} \mathrm{O}_{4} / \mathrm{PEI} \mathrm{NPs} / \mathrm{mL}(\mathrm{p}<0.001)$. Cell viability of different cell types, such as A3 human T lymphocytes and Sprague-Dawley rat smooth muscle cells, are also not 
affected after 24 hours incubation at low magnetic NPs concentrations (5-100 $\mu$ g magnetic NPs/mL), with reduced viability at higher concentrations (Ying, Hwang, 2010; Zhang, Chen et al., 2008). Hence, due to this detected dose-dependent cytotoxicity, higher concentrations than $80 \mu \mathrm{g} \mathrm{Fe} \mathrm{O}_{4} / \mathrm{PEI} \mathrm{NPs} / \mathrm{mL}$ were not used in the following experiments.

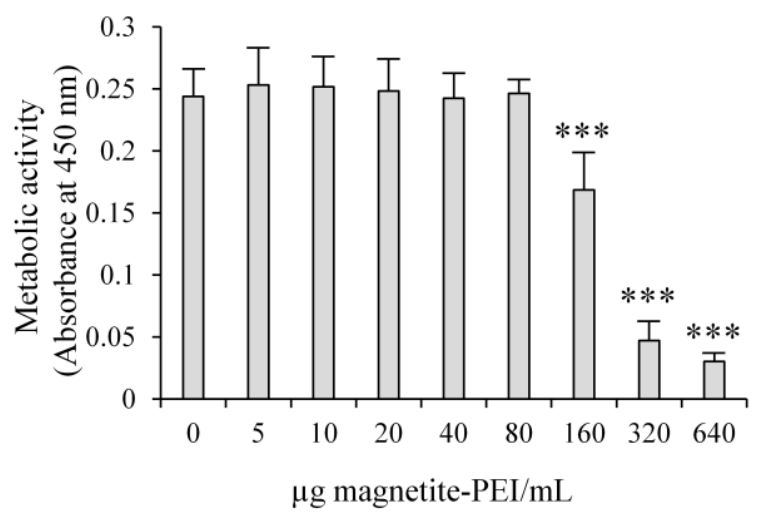

Figure 5. Metabolic activity of INS1E pseudoislets after incubation with different concentrations of $\mathrm{Fe}_{3} \mathrm{O}_{4} / \mathrm{PEI}$ nanoparticles for $24 \mathrm{~h}$. Values represent mean \pm SD.***:p $<0.001$ compared to control incubated without $\mathrm{Fe}_{3} \mathrm{O}_{4} / \mathrm{PEI}$ nanoparticles.

The magnetic NPs concentration used in the magnetization step should be enough to give the pseudoislets sufficient magnetic properties to allow the displacement of the microcapsules during the purification process. Using 40 and $80 \mu \mathrm{g} \mathrm{Fe}_{3} \mathrm{O}_{4} / \mathrm{PEI} \mathrm{NPs} / \mathrm{mL}$, appropriate magnetic properties were provided to pseudoislets, since motion was detected when a magnetic field was applied, by placing a magnet next to the petri dish, with higher mobility of the pseudoislets that had been incubated with $80 \mu \mathrm{g} \mathrm{Fe}_{3} \mathrm{O}_{4} / \mathrm{PEI}$ NPs/mL. Likewise, in other studies, porcine pancreatic islets have been magnetized with $100 \mu \mathrm{g}$ magnetic NPs/mL without affecting their viability and being magnetically directed as desired when a magnetic field was applied. Also, they had been imaged and tracked when implanted in vivo by magnetic resonance imaging (RMI) (Kennedy et al., 2007; Kim, Kim et al., 2010). In our studies, both 40 and $80 \mu \mathrm{g} \mathrm{Fe}_{3} \mathrm{O}_{4} / \mathrm{PEI} \mathrm{NPs} / \mathrm{mL}$ concentrations were able to confer magnetic motion to the pseudoislets. However, after microencapsulation, the magnetized pseudoislets should be able to displace the whole microcapsule through the microfluidic device towards 
the magnetic channel. Regarding the biosafety of the use of SPIONs, it is well known that, at appropriate concentrations, they do not display cytotoxic effects; besides, magnetic nanoparticles are metabolized in the lysosomes after intracellular uptake and used in the production of hemoglobin and transferrin becoming part of the normal iron metabolism pathway of the body (Thakor, Jokerst et al., 2016). Therefore, in our approach, the magnetic nanoparticles inside the microcapsules will not have any contact with the surrounding tissue at the implantation site and will be metabolized by the encapsulated pseudoislets, thereby ensuring great biosafety.

Overall, since the $80 \mu \mathrm{g} \mathrm{Fe}_{3} \mathrm{O}_{4} / \mathrm{PEI} \mathrm{NPs} / \mathrm{mL}$ concentration demonstrated to provide higher mobility to the pseudoislets, this concentration was chosen for the subsequent pseudoislets magnetization and purification processes.

\subsection{In vitro evaluation of microencapsulated pseudoislets after microfluidic purification}

Two different types of microcapsules for encapsulated magnetized pseudoislets were studied: alginate microcapsules (A) and alginate-poly-L-lysine-alginate (APA) microcapsules. Both types of microcapsules display different key physical properties, mechanical strength, and macromolecules diffusion, which are crucial for the microcapsules integrity during the magnetic sorting and the pseudoislet insulin release after purification. A microcapsules provide higher diffusion rates than APA microcapsules, since the PLL coating reduce the porosity of the microcapsule surface, thus potentially affecting the diffusion of the therapeutic molecules secreted by the microencapsulated cells such as insulin. However, the PLL coating in APA microcapsules confers higher mechanical strength (Shen, Mazumder et al., 2009; Wilson, Najia et al., 2014), a crucial characteristic that they need to fulfill in order to avoid their breakage during the high mechanical stress generated in the magnetic

purification process. Hence, A microcapsules are good candidates for pseudoislets 
microencapsulation in terms of better insulin diffusion, while APA microcapsules are good candidates in terms of higher mechanical stability.

After pseudoislets microencapsulation at a density of 2000 pseudoislets/mL of alginate, we performed the magnetic purification with three recircularization steps, collecting microcapsules from the magnetic channel (purified microcapsules). Samples from the nonpurified and purified microcapsules were evaluated under the brightfield microscope (Figure 6a-b). Many empty microcapsules were observed in the non-purified sample (Figure 6a), with a higher presence of microencapsulated pseudoislets in the purified sample (Figure 6b). It was evidenced that the pseudoislets featured the expected magnetic properties and enabled the motion of the microcapsules towards the magnets placed on the microfluidic device, allowing their purification. Besides, microcapsules endured the mechanical stress suffered during the purification process, keeping their spherical shape while maintaining intact the pseudoislets.

(a)

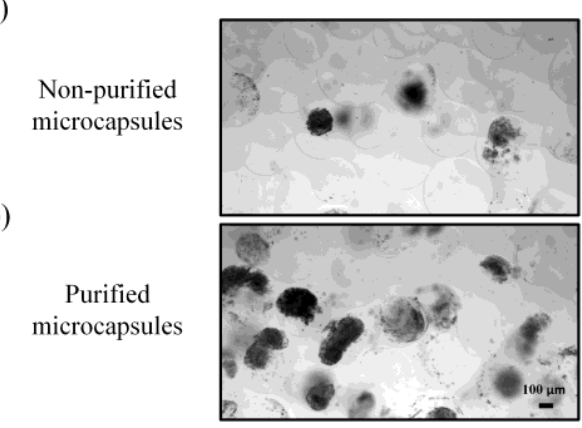

(c)

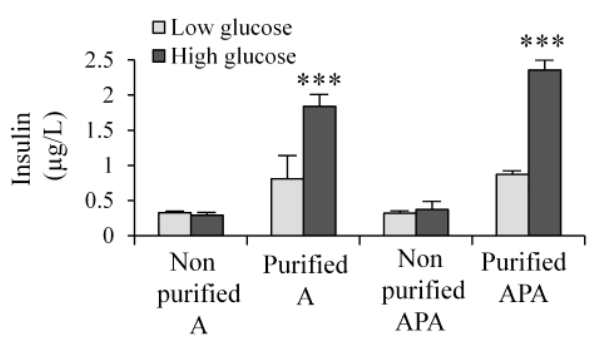

Figure 6. (a) Representative brightfield microphotographs of microencapsulated pseudoislets directly after encapsulation (Non-purified microcapsules) and (b) after 3 recircularized magnetic separations (Purified microcapsules). (c) Glucose-stimulated insulin secretion of A and APA microencapsulated INS1E pseudoislets before and after 3 recircularized magnetic separations. Values represent mean \pm SD.***:p<0.001 compared to low glucose condition. 
Next, the biological function of A and APA microencapsulated pseudoislets after purification was analyzed. For this purpose, the insulin secretory response to glucose challenges was evaluated after exposing non-purified and purified A and APA microcapsules to low and high glucose concentrations (3.3mM and $16.7 \mathrm{mM}$, respectively) (Figure 6c). For the non-purified samples, insulin levels were almost below the lower detection limit, and no significant differences were observed between low and high glucose conditions, probably due to the very low presence of microencapsulated pseudoislets. However, purified A and APA samples showed higher insulin levels. These results evidenced the capacity of both types of microcapsules for insulin production and release. Regarding the glucose responsiveness of the encapsulated pseudoislets, the secretion of insulin at high-glucose stimulus increased significantly compared to low-glucose stimulus $(\mathrm{p}<0.001)$ both in A and APA purified samples (Figure 5c), with similar insulin folding between high and low conditions: $2.52 \pm 0.52$ and 2,71 \pm 0.16 times more insulin secreted in high glucose than in low glucose conditions, for A and APA microcapsules, respectively. Therefore, purified $\mathrm{Fe}_{3} \mathrm{O}_{4} / \mathrm{PEI}$ NPs-conjugated pseudoislets maintained their capacity to secrete insulin as well as the glucose responsiveness within both A and APA microcapsules after the purification process.

We also quantified the insulin secretion from purified and non-purified A and APA microcapsules over the time, which allowed to estimate the purification efficiency just after purifying and assess the evolution of insulin production over time for 28 days. The insulin secretion ratio (purified/non-purified samples) of each time point were compared between $\mathrm{A}$ and APA microencapsulated pseudoislets (Figure 7a). Comparing these ratios, at day 1, similar insulin folding for A and APA microcapsules were obtained, $4.43 \pm 0.59$ and $4.67 \pm 0.9$ respectively, which entails a volume reduction of $77.5 \%$ for A microcapsules and, $78.6 \%$ for APA microcapsules with respect to each non-purified sample. The values for APA microcapsules remained stable with average insulin secretion ratio values between 4.2-4.6 
during the length of the study, but the insulin ratio from A microcapsules decreased during the first three weeks compared to APA microcapsules; from initial average insulin ratios of 4.4 to final values of 3.2. Comparing both groups, we detected statistically significant differences at the end of the study, when the ratio values from A microcapsules were lower than those from APA microcapsules $(\mathrm{p}<0.01$, at day 20 , and $\mathrm{p}<0.05$, at day 28). These differences between purified/non-purified A and APA microcapsules insulin ratios correlated with their metabolic activity, where purified A microcapsules showed significant lower metabolic activity values at day 28 compared to APA microcapsules $(\mathrm{p}<0.01)$ (Figure 7b). To explain these results, microcapsules physical integrity and cell viability of A and APA purified samples were analyzed over time under brightfield and fluorescence microscope (Figure 7c). Pseudoislets from both types of microcapsules showed cell death at day 1 on the surface of the pseudoislets due to the mechanical stress suffered during the microencapsulation process. The analysis of A microcapsules under brightfield displayed some unencapsulated small cell aggregates at day 1, which were more abundant at the end of the study (day 21 and 28 after purification). Moreover, the microscopy analysis unveiled that the pseudoislets growth inside A microcapsules provoked an excessive internal mechanical stress that led to the progressive breakage of these microcapsules. This progressive A microcapsules rupture released pseudoislets and, subsequently, the unprotected pseudoislets fragmented into smaller cell aggregates due to the mechanical stress when manipulated for microscopy analysis. Increasing amounts of fragmented pseudoislets were observed from day 1 till the end of the study. In contrast, in APA microcapsules samples, aggregates were not detected in the media; instead, all pseudoislets remained microencapsulated and, importantly, no evidence of microcapsules breakage was observed all over time. The higher mechanical strength of APA microcapsules, therefore, allowed restricting the pseudoislets growth, maintaining the pseudoislets within the matrix, thus improving the biosafety of the graft. The 
reduced mechanical integrity of A microcapsules can be attributed to the poor stability of the reversible ionic crosslinking of the sodium alginate macromolecules with a divalent ion, such as for example $\mathrm{Ca} 2+$ ions. In fact, the gelled alginate can exchange $\mathrm{Ca} 2+$ ions with the $\mathrm{Na}+$ present in the media, leading to a progressive degradation of the alginate hydrogels, which is an interesting property for cell delivery applications, where cells are required to escape from the microcapsule (Wilson et al., 2014). In contrast, APA microcapsules are protected against osmosis by the PLL coating, which strengthens the microcapsule, preventing its swelling and loss of stiffness (Shen et al., 2009; Wilson et al., 2014). Therefore, the decrease of the purified/non-purified insulin release and metabolic activity ratios for the A microcapsules compared to the APA microcapsules might occur due to the differences on their mechanical integrity that led to the loss of A microencapsulated pseudoislets. Importantly, for in vivo application in T1DM reversal, microcapsules need to hold physical and osmotic stress to avoid any cell exposure (Opara, Mirmalek-Sani et al., 2010; Vaithilingam, Tuch, 2011), since the breakage of the microcapsules may trigger the host's immune rejection against the exposed pseudoislets, implying the graft failure. Based on these results, APA microcapsules were selected in order to perform the implantation of pseudoislets into STZ-induced diabetic Wistar rats. 
(a)

(c)

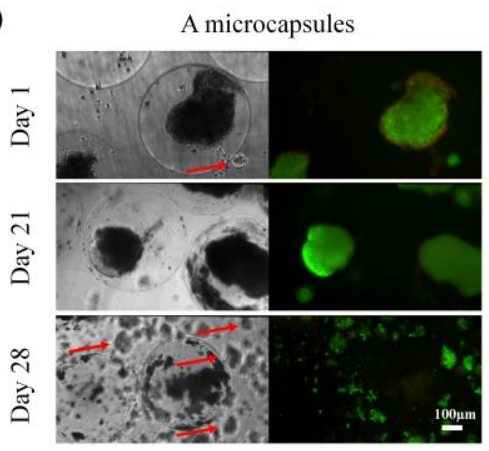

(b)
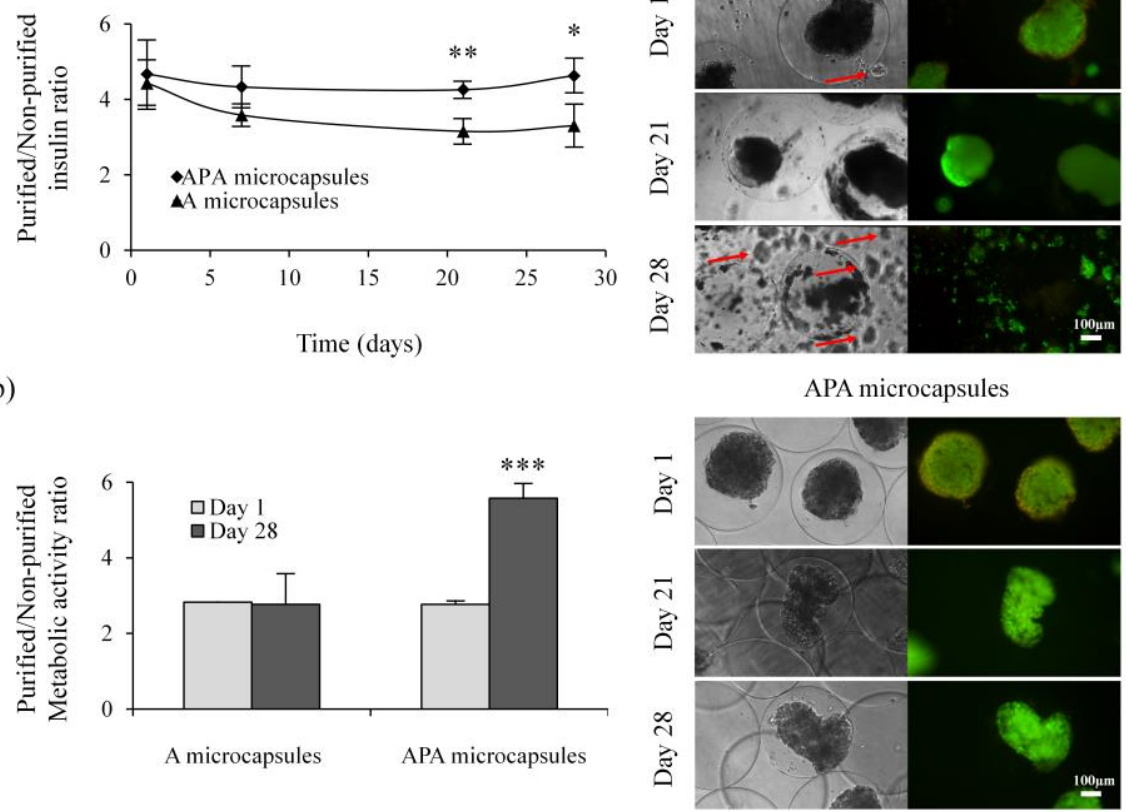

Figure 7. Purified/non-purified ratios of (a) insulin production and (b) metabolic activity, of A and APA microencapsulated INS1E pseudoislets before and after magnetic separation. Values represent mean $\pm \mathrm{SD}: * \mathrm{p}<0.05, * *: \mathrm{p}<0.01$ and $* * *: \mathrm{p}<0.001$. (c) Brightfield microphotographs and fluorescence microscopy images of purified A and APA microencapsulated INS1E pseudoislets (green fluorescence for live cells and red for dead cells). Red arrows identify fragmented INS1E cell aggregates. Scale bar: $100 \mu \mathrm{m}$.

\subsection{Normoglycemia restoration of STZ-induced diabetic Wistar rats}

Blood glucose levels of all studied groups (see 3.9 section for details of animal groups) were monitored for 142 days after STZ injection (Figure 8a). During the first 11 days after implantation of $0.4 \mathrm{~mL}$ of microcapsules from purified, non-purified and empty microcapsules pools, rats from all STZ-treated groups remained diabetic, with blood glucose levels between 350-500mg/dL, with no significant differences among groups. In contrast, non-diabetic control rats maintained their blood glucose levels around 100mg/dL. During the whole study, rats from the diabetic group and those implanted with non-purified microcapsules and empty microcapsules maintained high glucose levels, between $400-500 \mathrm{mg} / \mathrm{dL}$, with no significant differences among them. However, 19 days after implantation, blood glucose levels of rats implanted with purified microcapsules significantly decreased, reaching values between 140$200 \mathrm{mg} / \mathrm{dL}$, which are comprised within the normal glycemic range $(<200 \mathrm{mg} / \mathrm{dL})$, very close 
to the non-diabetic control levels. These results correlated with the bodyweight gain and cumulative survival analysis data (Figure 8b-c). Diabetic control animals did not gain weight during the first weeks, begun to show discomfort 22 days after implantation and, subsequently, were sacrificed. Similarly, rats implanted with non-purified and empty microcapsules did not gain weight during the first weeks either, but discomfort appeared later, from day 44 to 66 after implantation. In contrast, rats implanted with purified microencapsulated pseudoislets began to gain weight 2 days after implantation, showing statistically significant higher body weight values during all the study compared to the rest of the diabetic groups $(\mathrm{p}<0.001)$. Non-diabetic control rats also gained weight during the study always showing statistically significant higher values than the rats implanted with purified microcapsules ( $\mathrm{p}<0.001)$, and all the animals survived the whole procedure, as expected.

(a)

(b)

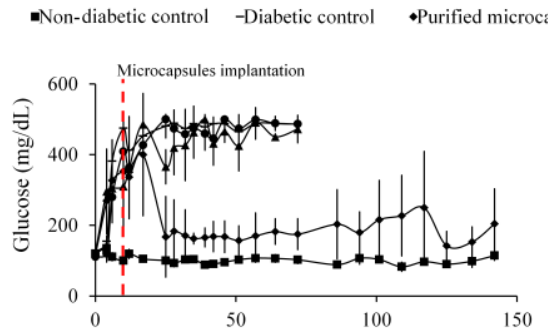

(c)

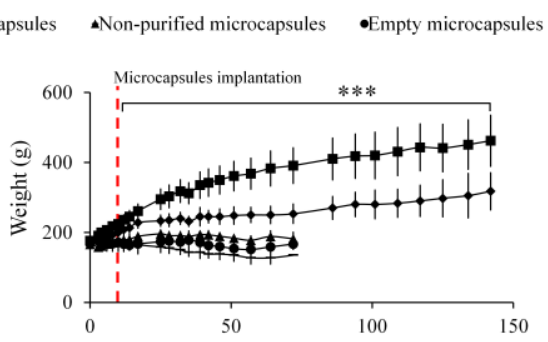

(d)

Days after STZ injection
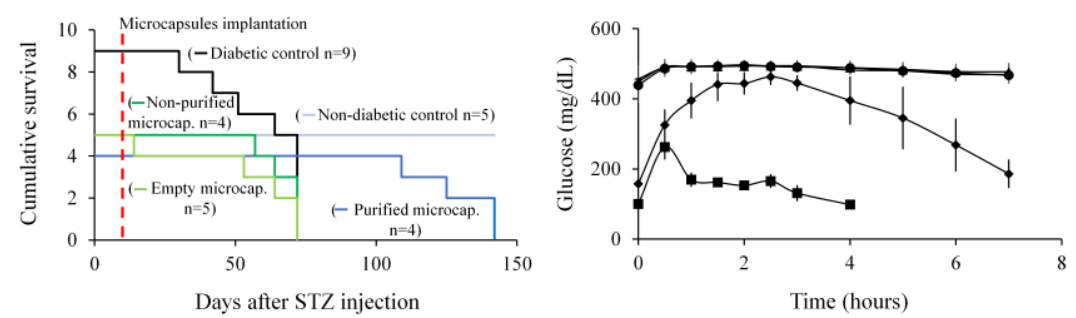

Figure 8. Long-term monitoring of blood glucose levels (a) and body weights (b) of STZ-induced diabetic Wistar rats implanted with empty microcapsules, non-purified and purified microcapsules containing INS1E pseudoislets. Non-diabetic and non-implanted diabetic rats were used as controls. (c) Intraperitoneal glucose tolerance test two months after microcapsules implantation. (d) Kaplan-Meier analysis of rats from different groups. Dotted red lines identify the microcapsules implantation time point. $* * *: p<0.001$ compared to rats implanted with purified microencapsulated pseudoislets. 
Focusing on the animals implanted with purified microcapsules, symptoms of graft failure were noticed on day 104 after implantation, being the last graft failure on day 136 post implantation. Similarly, Albino Oxford (AO) rats implanted with 2-2.5mL of non-purified APA microencapsulated allogeneic Lewis-islets become normoglycemic within 5 days after implantation and, remained normoglycemic, with blood glucose levels below 200mg/dL. However, some animals began to show symptoms of graft failure 42 days after implantation (Paul de Vos, van Hoogmoed et al., 2003). Therefore, although the initial therapeutic effect of our purified microencapsulated pseudoislets was detected later than in the study by de Vos and cols (Paul de Vos et al., 2003), our implants demonstrated better results in terms of normoglycemia maintenance. In fact, AO rats showed the first symptoms of graft failure just 42 days after implantation, and in our study, the first graft failure was detected on day 104 . Hence, these data demonstrate that implantation of $0.4 \mathrm{~mL}$ of purified microencapsulated pseudoislets is able to restore blood glucose levels within the normoglycemic range. In contrast, in other studies, implantation volumes of microencapsulated allogeneic pancreatic islets have been ranged between $2-2.5 \mathrm{~mL}$ per animal in order to achieve normoglycemia in STZ-induced diabetic AO rats (P. De Vos, Van Straaten et al., 1999; Paul de Vos et al., 2003). Therefore, we have achieved blood glucose levels restoration into the normoglycemic range with a 5 to 6.25 times reduction in the implant volume. Undoubtedly, this is a highly relevant achievement for reducing the host's immune response against the graft (King, 2001).

In order to evaluate the capacity of the different animal groups to respond to glucose stimuli, we performed a glucose tolerance test two months after implantation (Figure 8d). As expected, results for the diabetic control animals and those implanted with non-purified and empty microcapsules showed no response to glucose stimulus, with high blood glucose levels around $500 \mathrm{mg} / \mathrm{dL}$. In contrast, in rats implanted with purified microcapsules, which showed initial blood glucose levels under $200 \mathrm{mg} / \mathrm{dL}$, glucose values increased up to $420-450 \mathrm{mg} / \mathrm{dL}$, 
smoothly decreasing to final values around $200 \mathrm{mg} / \mathrm{dL} 7$ hours after the glucose administration. Non-diabetic control rats showed a stronger response to glucose stimulus with lower peak values (around 230mg/dL), requiring less time for normoglycemic glucose values restoration, which occurred 2 hours after glucose administration. Similarly to our results, AO rats implanted with microencapsulated allogeneic Lewis-islets show higher initial blood glucose levels than non-diabetic control in the glucose tolerance test. However, in that study, animals are able to diminish blood glucose levels faster than our rats implanted with purified microcapsules (Paul de Vos et al., 2003). This difference might be due to graft implantation site. In fact, in type I diabetes mellitus patients, insulin is detected faster in the bloodstream when it is administrated through intraperitoneal injection than when it is administered subcutaneously, with ranges between 60-150 minutes and 150-300 minutes, respectively (Giacca, Caumo et al., 1993). This occurs due to the different degree of vascularization of the implantation site; the high vascularization of the peritoneal cavity promotes faster insulin absorption, while the subcutaneous tissue is not that highly vascularized, limiting the diffusion of insulin towards the bloodstream (Burnett, Huyett et al., 2014; Figliuzzi, Cornolti et al., 2005). This could have been the reason why in our subcutaneously implanted rats we noticed a delayed glucose response and slower restoration of normoglycemia. Nevertheless, although the implantation of microencapsulated islets in the peritoneum cavity has shown faster glucose response in $\mathrm{AO}$ rats, this location has several disadvantages that make it not suitable for clinical application. One important one is that the implantation of microcapsules in this location goes through an invasive surgical technique which provokes a strong inflammatory response in the implantation site (P. De Vos et al., 1999; Robitaille, Dusseault et al., 2005). In addition, the high vascularization of the peritoneum facilitates the easy access of the host's immune cells, which transforms the acute inflammatory reaction into a chronic process that leads to the graft failure (Robitaille et al., 2005). Finally, in the peritoneum, 
microencapsulated islets are freely floating, which would difficult the removal of the whole graft if required, compromising the biosafety of the implant. Unfortunately, this situation would force the use of more invasive techniques such as peritoneum lavage (P. De Vos et al., 1999; Paul de Vos et al., 2003).

Lastly, we performed a histological evaluation by hematoxylin and eosin and Masson's trichrome staining in order to examine the retrieved microcapsules and to evaluate the inflammatory response (Figure 9a-b). Collagen-like surrounding tissue was detected, indicating the presence of fibrotic tissue in all the samples, with no differences among the different grafts, independently of the implanted microcapsules (Figure 9a). Also, no differences among groups were detected on the surrounding inflammatory response with the presence of some infiltrating lymphocyte and neutrophil cells. Regarding the microcapsules, in the empty and non-purified microcapsules samples, we mainly observed empty microcapsules, while in the purified sample, we observed a higher presence of microencapsulated pseudoislets without cell protruding (Figure 9b). Importantly, although the purified sample contained higher quantities of pseudoislets, the inflammatory response was similar to the empty and non-purified microcapsules samples, since the pseudoislets from the purified group remained encapsulated and did not trigger higher host's immune response.

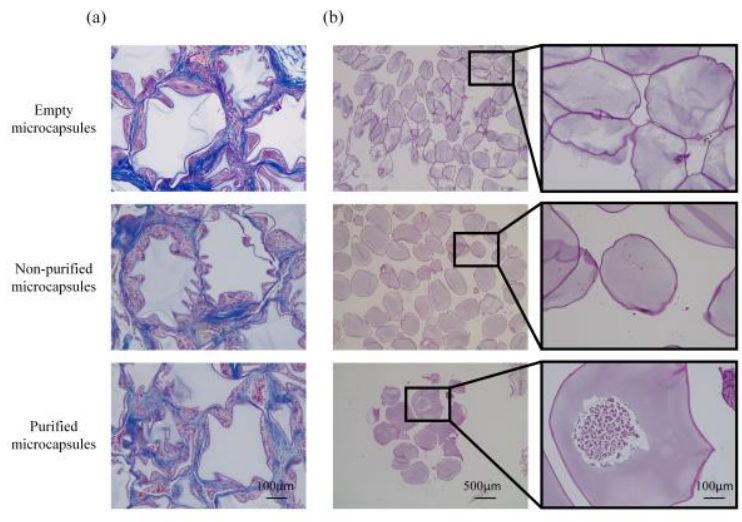

Figure 9. Foreign body reaction analysis of subcutaneously implanted empty microcapsules, non-purified and purified microencapsulated INS1E pseudoislets in STZ-induced diabetic Wistar rats. (a) Representative photographic images of Masson's trichrome staining of explanted grafts. (b) Representative images of hematoxylin-eosin staining of explanted grafts. Scale bars: $100 \mu \mathrm{m}$ or $500 \mu \mathrm{m}$. 


\section{Conclusions}

The combination of 3D printing, microfluidics, magnetic sorting, and magnetic cell labeling technologies, enabled the production of a magnetic sorting device for the purification of magnetically labeled encapsulated pseudoislets. While these implants showed the capacity to normalize glucose blood levels in diabetic rats, a considerable reduction of the graft volume has been accomplished (higher than 75\%), compared to previously reported works. Our microfluidic device provides high purification yields, enables the monitorization of the process and avoids manual steps, thus, minimizing technical errors and improving the reproducibility of the purification process. Moreover, the miniaturized nature of the approach facilitates the parallelization of processes, the multiplexing capabilities, and high-throughput screening. Therefore, this technology will improve the efficacy of therapeutic strategies that include the use of microencapsulated pancreatic islets for the Type 1 Diabetes Mellitus clinical management. In this sense, future directions for its widespread clinical application should focus on scaling-up the procedure. On this regard, further investigations on the development of a technology that is able to perform the microencapsulation and the sorting processes in one single step would suppose a step forward in the optimization and reduction of the costs of this approach, bringing this technology closer to the clinics.

\section{Acknowledgments}

Authors thank the support to research from the University of the Basque Country UPV/EHU (EHUa16/06 to L.SB, and ESPPOC 16/65), the Basque Country Government (Grupos Consolidados with Grant $\mathrm{N}^{\mathrm{o}}$ IT907-16 to JL. P, Elkartek with Grant $\mathrm{N}^{\mathrm{o}} \mathrm{KK}$ 2017/0000088 and RIS3 with Grant N³07616FKA4) and the Spanish Government (RYC2012-10796). Authors also wish to thank the intellectual and technical assistance from the ICTS "NANBIOSIS", more specifically by the Drug Formulation Unit (U10) of the CIBER in Bioengineering, Biomaterials \& Nanomedicine (CIBER-BBN) at the University of Basque 
Country UPV/EHU, Prof. Maechler from the University of Geneva Medical Center for providing the INS1E cell line, Adhesive Research for providing PSA sheets and Prof. Martínez de Pancorbo for her laboratory facilities at University of the Basque Country UPV/EHU.

\section{References}

Adeyemi, A. H. (2017). Microfluidic devices for the characterization and manipulation of encapsulated cells in agarose microcapsules using dielectrophoresis and electrophoresis. Thesis. Faculty of Engineering. UNIVERSITY OF OTTAWA.

Ahne Myklatun, Michele Cappetta, Michael Winklhofer, Vasilis Ntziachristos, \& Gil G Westmeyer. (2017). Microfluidic sorting of intrinsically magnetic cells under visual control. Scientific Reports (Nature Publisher Group), 7, 1. doi:10.1038/s41598-017-06946-X

Borg, D. J., \& Bonifacio, E. (2011). The use of biomaterials in islet transplantation. Current Diabetes Reports, 11(5), 434-444. doi:10.1007/s11892-011-0210-2

Brian Buder, Michael Alexander, Rahul Krishnan, David W Chapman, \& Jonathan RT Lakey. (2013). Encapsulated islet transplantation: Strategies and clinical trials. Immune Network, 13(6), 235-239. doi:10.4110/in.2013.13.6.235

Burnett, D. R., Huyett, L. M., Zisser, H. C., Doyle, F. J., \& Mensh, B. D. (2014). Glucose sensing in the peritoneal space offers faster kinetics than sensing in the subcutaneous space. Diabetes, 63(7), 2498-2505. doi:10.2337/db13-1649

de Groot, M., Schuurs, T. A., \& van Schilfgaarde, R. (2004). Causes of limited survival of microencapsulated pancreatic islet grafts. Journal of Surgical Research, 121(1), 141-150.

De Vos, P., Van Straaten, J. F., Nieuwenhuizen, A. G., de Groot, M., Ploeg, R. J., De Haan, B. J., et al. (1999). Why do microencapsulated islet grafts fail in the absence of fibrotic overgrowth? Diabetes, 48(7), 1381-1388.

de Vos, P., \& Marchetti, P. (2002). Encapsulation of pancreatic islets for transplantation in diabetes: The untouchable islets. Trends in Molecular Medicine, 8(8), 363-366. doi:10.1016/S1471-4914(02)02381-X

de Vos, P., van Hoogmoed, C. G., van Zanten, J., Netter, S., Strubbe, J. H., \& Busscher, H. J. (2003). Long-term biocompatibility, chemistry, and function of microencapsulated pancreatic islets doi://doi.org/10.1016/S0142-9612(02)00319-8

Dressaire, E., \& Sauret, A. (2016). Clogging of microfluidic systems. Soft Matter, 13(1), 3748. doi:10.1039/c6sm01879c 
Edmond A. Ryan, Jonathan R.T. Lakey, Ray V. Rajotte, Gregory S. Korbutt, Tatsuya Kin, Sharleen Imes, et al. (2001). Clinical outcomes and insulin secretion after islet transplantation with the edmonton protocol. Diabetes, 50(4), 710-719. doi:10.2337/diabetes.50.4.710

El-Sherbiny, I. M., \& Yacoub, M. H. (2013). Hydrogel scaffolds for tissue engineering: Progress and challenges. Global Cardiology Science \& Practice, 2013(3), 316-342. doi:10.5339/gcsp.2013.38

Figliuzzi, M., Cornolti, R., Plati, T., Rajan, N., Adobati, F., Remuzzi, G., et al. (2005). Subcutaneous xenotransplantation of bovine pancreatic islets doi://doi.org/10.1016/j.biomaterials.2005.02.019

Giacca, A., Caumo, A., Galimberti, G., Petrella, G., Librenti, M. C., Scavini, M., et al. (1993). Peritoneal and subcutaneous absorption of insulin in type I diabetic subjects. The Journal of Clinical Endocrinology and Metabolism, 77(3), 738-742. doi:10.1210/jcem.77.3.8370695

Girault, M., Kim, H., Arakawa, H., Matsuura, K., Odaka, M., Hattori, A., et al. (2017). An on-chip imaging droplet-sorting system: A real-time shape recognition method to screen target cells in droplets with single cell resolution. Scientific Reports, 7, 40072. doi: $10.1038 /$ srep40072

Ho, C. M., Ng, S. H., Li, K. H., \& Yoon, Y. J. (2015). 3D printed microfluidics for biological applications. Lab on a Chip, 15(18), 3627-3637. doi:10.1039/c5lc00685f

Kennedy, D. J., Todd, P., Logan, S., Becker, M., Papas, K. K., \& Moore, L. R. (2007). Engineering quadrupole magnetic flow sorting for the isolation of pancreatic islets doi://doi.org/10.1016/j.jmmm.2006.10.1160

Kim, H. S., Kim, H., Park, K. S., \& Moon, W. K. (2010). Evaluation of porcine pancreatic islets transplanted in the kidney capsules of diabetic mice using a clinically approved superparamagnetic iron oxide (SPIO) and a 1.5T MR scanner. Korean Journal of Radiology, 11(6), 673-682. doi:10.3348/kjr.2010.11.6.673

King, A. (2001). Microencapsulation of islets of langerhans: Impact of cellular overgrowth. Upsala Journal of Medical Sciences, 106(3), 161-174.

Kobayashi, T., Aomatsu, Y., Iwata, H., Kin, T., Kanehiro, H., Hisanga, M., et al. (2006). Survival of microencapsulated islets at 400 days posttransplantation in the omental pouch of NOD mice. Cell Transplantation, 15(4), 359-365. doi:10.3727/000000006783981954

Lee, K. Y., \& Mooney, D. J. (2011). Alginate: Properties and biomedical applications. Progress in Polymer Science, 37(1), 106. doi:10.1016/j.progpolymsci.2011.06.003

Li, P., Tian, Y., \& Pappas, D. (2011). Comparison of inlet geometry in microfluidic cell affinity chromatography. Analytical Chemistry, 83(3), 774-781. doi:10.1021/ac102975g

Megías, R., Arco, M., Ciriza, J., Burgo, L. S. d., Puras, G., López-Viota, M., et al. (2017). Design and characterization of a magnetite/PEI multifunctional nanohybrid as non-viral 
vector and cell isolation system. International Journal of Pharmaceutics, 518(1-2), 270-280. doi:10.1016/j.ijpharm.2016.12.042

Merglen, A., Theander, S., Rubi, B., Chaffard, G., Wollheim, C. B., \& Maechler, P. (2004). Glucose sensitivity and metabolism-secretion coupling studied during two-year continuous culture in INS-1E insulinoma cells. Endocrinology, 145(2), 667-678. doi:10.1210/en.20031099

Muñoz de Escalona, M., Sáez-Fernández, E., Prados, J. C., Melguizo, C., \& Arias, J. L. (2016). Magnetic solid lipid nanoparticles in hyperthermia against colon cancer. International Journal of Pharmaceutics, 504(1-2), 11-19. doi:10.1016/j.ijpharm.2016.03.005

Opara, E. C., Mirmalek-Sani, S. H., Khanna, O., Moya, M. L., \& Brey, E. M. (2010). Design of a bioartificial pancreas(+). Journal of Investigative Medicine : The Official Publication of the American Federation for Clinical Research, 58(7), 831-837. doi:10.231/JIM.0b013e3181ed3807

Park, H., Lee, H. J., An, H., \& Lee, K. Y. (2017). Alginate hydrogels modified with low molecular weight hyaluronate for cartilage regeneration. Carbohydrate Polymers, 162, 100107. doi:10.1016/j.carbpol.2017.01.045

Robitaille, R., Dusseault, J., Henley, N., Desbiens, K., Labrecque, N., \& Hallé, J. (2005). Inflammatory response to peritoneal implantation of alginate-poly-l-lysine microcapsules doi://doi.org/10.1016/j.biomaterials.2004.10.028

Ryan, E. A., Lakey, J. R. T., Paty, B. W., Imes, S., Korbutt, G. S., Kneteman, N. M., et al. (2002). Successful islet transplantation: Continued insulin reserve provides long-term glycemic control. Diabetes, 51(7), 2148-2157. doi:10.2337/diabetes.51.7.2148

Sakata, N., Sumi, S., Yoshimatsu, G., Goto, M., Egawa, S., \& Unno, M. (2012). Encapsulated islets transplantation: Past, present and future. World Journal of Gastrointestinal Pathophysiology, 3(1), 19-26. doi:10.4291/wjgp.v3.i1.19

Shen, F., Mazumder, M. A., Burke, N. A., Stover, H. D., \& Potter, M. A. (2009). Mechanically enhanced microcapsules for cellular gene therapy. Journal of Biomedical Materials Research.Part B, Applied Biomaterials, 90(1), 350-361. doi:10.1002/jbm.b.31292

Skrzypek, K., Groot Nibbelink, M., Lente, J. v., Buitinga, M., Engelse, M. A., Koning, E. J. d., et al. (2017). Pancreatic islet macroencapsulation using microwell porous membranes. Scientific Reports, 7, 9186. doi:10.1038/s41598-017-09647-7

Smidsrod, O., \& Skjak-Braek, G. (1990). Alginate as immobilization matrix for cells. Trends in Biotechnology, 8(3), 71-78.

Strand, B. L., Ryan, T. L., In't Veld, P., Kulseng, B., Rokstad, A. M., Skjak-Brek, G., et al. (2001). Poly-L-lysine induces fibrosis on alginate microcapsules via the induction of cytokines. Cell Transplantation, 10(3), 263-275. 
Street, C. N., Lakey, J. R. T., Shapiro, A. M. J., Imes, S., Rajotte, R. V., Ryan, E. A., et al. (2004). Islet graft assessment in the edmonton protocol: Implications for predicting long-term clinical outcome. Diabetes, 53(12), 3107-3114. doi:10.2337/diabetes.53.12.3107

Temiz, Y., Lovchik, R. D., Kaigala, G. V., \& Delamarche, E. (2015). Lab-on-a-chip devices: How to close and plug the lab? doi://doi.org/10.1016/j.mee.2014.10.013

Tendulkar, S., Mirmalek-Sani, S. H., Childers, C., Saul, J., Opara, E. C., \& Ramasubramanian, M. K. (2012). A three-dimensional microfluidic approach to scaling up microencapsulation of cells. Biomedical Microdevices, 14(3), 461-469. doi:10.1007/s10544011-9623-6

Thakor, A. S., Jokerst, J. V., Ghanouni, P., Campbell, J. L., Mittra, E., \& Gambhir, S. S. (2016). Clinically approved nanoparticle imaging agents. Journal of Nuclear Medicine: Official Publication, Society of Nuclear Medicine, 57(12), 1833-1837.

Vaithilingam, V., \& Tuch, B. E. (2011). Islet transplantation and encapsulation: An update on recent developments. The Review of Diabetic Studies: RDS, 8(1), 51-67. doi:10.1900/RDS.2011.8.51

Wanyu Chen, Mark Lisowski, Gamal Khalil, Ian R Sweet, \& Amy Q Shen. (2012). Microencapsulated 3-dimensional sensor for the measurement of oxygen in single isolated pancreatic islets. PLoS One, 7(3), e33070. doi:10.1371/journal.pone.0033070

Wenjuan Li, Ruxing Zhao, Jidong Liu, Meng Tian, Yiran Lu, Tianyi He, et al. (2014). Small islets transplantation superiority to large ones: Implications from islet microcirculation and revascularization. Journal of Diabetes Research, 2014, 192093. doi:10.1155/2014/192093

Wilson, J. L., Najia, M. A., Saeed, R., \& McDevitt, T. C. (2014). Alginate encapsulation parameters influence the differentiation of microencapsulated embryonic stem cell aggregates. Biotechnology and Bioengineering, 111(3), 618-631. doi:10.1002/bit.25121

Wyatt Shields IV, C., Reyes, C. D., \& López, G. P. (2015). Microfluidic cell sorting: A review of the advances in the separation of cells from debulking to rare cell isolation. Lab on a Chip, 15(5), 123-1249. doi:10.1039/c4lc01246a

Xi, H., Zheng, H., Guo, W., Gañán-Calvo, A. M., Ai, Y., Tsao, C., et al. (2017). Active droplet sorting in microfluidics: A review. Lab Chip, 17(5), 751-771. doi:10.1039/c6lc01435f

Yang, H. K., \& Yoon, K. H. (2015). Current status of encapsulated islet transplantation. Journal of Diabetes and its Complications, 29(5), 737-743. doi:10.1016/j.jdiacomp.2015.03.017

Ying, E., \& Hwang, H. (2010). In vitro evaluation of the cytotoxicity of iron oxide nanoparticles with different coatings and different sizes in A3 human $T$ lymphocytes doi://doi.org/10.1016/j.scitotenv.2010.07.025

Yun Lee, D., Hee Nam, J., \& Byun, Y. (2007). Functional and histological evaluation of transplanted pancreatic islets immunoprotected by PEGylation and cyclosporine for 1 year. Biomaterials, 28(11), 1957-1966. doi:10.1016/j.biomaterials.2006.12.015 
Zhang, S., Chen, X., Gu, C., Zhang, Y., Xu, J., Bian, Z., et al. (2008). The effect of iron oxide magnetic nanoparticles on smooth muscle cells. Nanoscale Research Letters, 4(1), 70. doi:10.1007/s11671-008-9204-7 